\title{
A reconciliation of dynamic crack velocity and Rayleigh wave speed in isotropic brittle solids
}

\author{
P.D. WASHABAUGH ${ }^{1}$ and W.G. KNAUSS ${ }^{2}$ \\ ${ }^{1}$ Department of Aerospace Engineering, University of Michigan, Ann Arbor, Michigan, 48109-2118, USA \\ ${ }^{2}$ Graduate Aeronautics Laboratories, California Institute of Technology, Pasadena, California, 91125, USA
}

Received 9 August 1993; accepted in final form 6 December 1993

\begin{abstract}
Following earlier observations of multiple micro-crack formation accompanying crack propagation under dynamic conditions, the question regarding the discrepancy between the 'theoretically anticipated' maximal crack (Rayleigh wave) speed and those observed typically in amorphous, isotropic solids is examined experimentally. It is shown that if the production of these multiple micro-cracks ahead of the main fracture is suppressed by fabricating a material possessing a thin uniform region of vanishing intrinsic (molecular/atomic) material strength, the crack speed is materially increased to the point of approaching the Rayleigh wave speed. Moreover, it is also shown that the presence of small discreet flaws of sufficient spatial density similarly 'weakens' the material to produce fracture speeds comparable to the Rayleigh wave speed. One deduces, therefore, that for a single crack front the linearized theory of elastodynamics correctly predicts the dynamic crack propagation behavior of a solid with sufficiently low material strength.
\end{abstract}

\section{Introduction}

The theory of elasticity has contributed many important aspects to the analysis and prediction of quasi-static and dynamic fracture. However, in spite of the extensive corroboration between experiments and analyses, one of the baffling disagreements in isotropic and homogeneous solids is the substantial discrepancy between the observed maximal speed of crack propagation [1] and the theoretically determined limit as the Rayleigh surface wave speed [2]. A number of explanations have been offered, some of which bear more phenomenological than physically specific character, varying from allusion to plasticity effects [3] and to material related rate dependence not experienced in the usual property characterizations [4]. This disagreement has generated a close examination of the limiting flow of energy into the crack tip region, partially associated with the question of crack branching, without however, resolving the issue of the high theoretical limit on the crack speed. In fact the limitation of sufficient energy flow into the moving crack-tip region makes the crack speed depend on the magnitude of the stress intensity factor such that the former approaches the Rayleigh wave velocity asymptotically. Experimental results tend to agree with the theoretical predictions except that they fall short of the expected limiting behavior by a factor of two or more [5].

Recently Gao [6] has explored the idea that the 'slower-than-theoretical' speed was the result of an 'unstable' sinusoidal path along which the actual crack speed equals the Rayleigh speed. While this proposition can result in average crack speeds (projected onto the average plane of crack propagation) which are on the order of half the Rayleigh wave speed, this upper limit conflicts with several experimental studies in which higher crack speeds are attained.* It appears

\footnotetext{
*In order to achieve a reduction in average crack speed of 50 percent of the Rayleigh wave speed in this oscillatory manner the amplitude of the sinusoidal path must be about 0.4 of its length. There is no record of this phenomenon in the experimental fracture literature.
} 
to make more sense to allow discontinuous processes to be associated with the reduction in the crack speed from the 'ideal' case. We refer here for example, to the studies by Field [7] and point out, in particular, that such sinusoidal crack motion contradicts the experimental results by Knauss and Lee [8] and those delineated here later on.

In contrast to explanations offered in the past we follow here the hypothesis that the (linearized) theory of elasticity does not incorporate any effect of intrinsic material strength and is thus unable to distinguish between phenomena associated with low (atomic or molecular) strength of a material as opposed to others for which this property has (relatively) high values. In a previous series of papers on experimental investigations of dynamic fracture [9] it has been pointed out that in an amorphous, brittle solid (Homalite 100, a polyester) fracture propagation was associated with multiple flaws (e.g. voids) or micro-cracks preceding the main crack, and that these multiple cracks coalesced to form the main crack. The number and size of these cracks depends on the magnitude of the stresses (i.e. stress intensity factor) at the crack tip, and that these induced micro-cracks are also the source of crack branching when the crack tip stresses are of sufficient magnitude. In the context of the crack propagation process it is clear that the occurrence of such multiple cracks has a significant influence on the rate with which it can proceed: First, it takes time for waves to travel back and forth between the individual micro-fractures in order to coordinate them into forming the major crack; second, it requires additional time for the small cracks to grow (laterally). These phenomena are not usually accounted for in elasticity models.

Because it is difficult to analytically examine the hypothesis that the micro-cracks are indeed responsible for the 'slowing' of the crack from its 'theoretical' speed, it is of interest to examine it from an experimental point of view. Underlying any such study is the fact that the multiple cracks are caused by the high stresses in the crack tip vicinity. If these high stresses could be somehow reduced so that these micro-cracks are not formed, while still providing sufficient energy for crack propagation, the crack should propagate at higher speeds, and possibly at the Rayleigh wave speed.

Field has reported experiments [7] involving anisotropic crystals possessing preferred planes along which cracks can propagate with the relatively lowest energy expenditure and presumably low stress and strain level. He also found cracks to propagate with substantially higher speeds than in the more conventional engineering materials and approaching the Rayleigh surface wave speeds. We presume that this result is derived from the suppression of, or reduction in, the number of micro-cracks ahead of the main one. The partial draw back of this ingenious work is the question as to whether the anisotropy has a disproportionate effect in the physics of crack propagation, if not in the analysis.

The generation of micro-cracks, and thus, presumably, the retardation of crack propagation, may be demonstrated by generating a material with controllable cohesive strength (a weak plane) while maintaining all other physical properties. One would thus argue that a 'strong' material will generate multiple cracks and lead to slow crack growth, while a 'weak' material generates no secondary cracks and should, therefore, produce higher crack speeds more in agreement with the elasticity theory, which also assumes that only a single crack front exists. Certainly, in the limit of vanishing material (molecular or atomic) strength there should be no limit to the rate of crack growth other than the Rayleigh wave speed, if the vanishing strength is responsible for the suppression of multiple micro-crack generation. 
This strength control can be achieved in uncrosslinked but 'brittle' polymers by fracturing a plate of the material cleanly and by then rehealing it to varying levels of strength through suitably controlled conditions. In this way the wave speeds of the material remain the same, while the forces that can be transmitted across the crack plane ahead of the tip are limited so as to prevent the formation of multiple cracks. The only question that remains open is whether the rehealing process generates conditions along the crack path that cause the stress and strain distributions across the fracture path to be different than they would be if the material was naturally one with a 'progressively weaker constitution'. This question may be answered, as argued later on, to a substantial degree by observing the deformation field at the running crack tip for any indicative or unusual phenomenon spanning the crack plane.

The argument that the interaction times between the major crack and the micro-flaws is responsible for the retardation in crack speeds elicits the thought that the mere existence of flaws along or surrounding the crack axis may be sufficient to influence crack growth velocities. A competing way of expressing this idea is that, perhaps, preexisting flaws are responsible for the 'retardation' of the crack. This is related to notions of a crack being shielded by some form of prior accumulated damage [10]. Flaws surrounding the crack could grow and lower the effective modulus of the material and thereby reduce the local effective wave speeds. Also, the flaws may act to blunt the propagating crack, thus promoting branching, and thereby reduce the energy available to advance the main crack. It is natural, therefore, to also investigate the influence of multiple discrete pre-existing flaws dispersed in some pattern in the material.

An early attempt in this direction of experimental examination has been documented by Knauss and Lee [8]. While that investigation clearly supported the notions regarding the role of material strength on the crack speed entertained above, the control over the strength was less than ideal. As a consequence, we re-examine this problem with a bonding technique that does not incorporate an additional adhesive material nor the use of solvents. In addition, the material response near the crack front will be examined to allow comparison between the pristine and processed materials.

We shall employ an uncrosslinked, brittle polymer for these studies, polymethylmethacrylate (PMMA) because it readily affords re-bonding without introducing an additional bonding agent; the latter could raise additional questions with regard to material constituents, regardless of the (possibly) small thickness one could impose on this layer. We believe that within time scales encountered under dynamic conditions PMMA may be considered an essentially brittle and largely rate insensitive material. If rate dependence were a crack-speed-reducing factor, it would, in light of the results shown later on, argue more strongly for the viability of the hypothesis advanced here, rather than against it. (Support of these notions is offered later on in this paper.)

In the sequel we discuss first, in Section 2, the method of specimen manufacture for producing variable cohesive strength, as well as introducing (drilling) small circular flaws along the anticipated crack path. In Section 3 the method of experimentally loading the specimens dynamically along with interferometer measurements, and image processing, are reviewed briefly. Section 4 delineates characteristic results, and the final section summarizes the results of this investigation. 


\section{Specimen preparation}

Three types of materials were employed. The first type consisted of plain sheets $30 \mathrm{~cm}$ square cast from PMMA (90000 molecular weight) supplied by Aldrich [11, 12]. The other two types of materials were nominally identical to the first except they contained a weakened interface. Weak 'planes' were incorporated into an otherwise amorphous material by two methods. In the first method the thermoplastic nature of the PMMA was exploited by imperfectly 'sintering' together two surfaces, the goal here being to perturb the otherwise pristine material with a thin plane of randomly distributed molecular imperfections. The second type of interface had a much larger scale and was produced by simply drilling a linear periodic array of holes, coplanar with the initial crack. All of these plates were nominally $4.5 \mathrm{~mm}$ thick.

\subsection{The 'sintered' interface}

A thin, uniform and enfeebled plane was introduced into an otherwise homogeneous plate by imperfectly sintering together two sheets. The intention here is to provide a weakened interface that (to a good approximation) can still be treated as a continuum. The temperature across the interface is used to control the rate of commingling or interpenetration of the polymer molecules. For sufficiently long times and temperatures, an interface can be completely rehealed, i.e. a seamless coupling between the two original plates. The sintered interface was produced and characterized as follows.

\subsubsection{Construction}

Two sheets were cut from a single previously cast plate. The edges of the two segments were finished with a high-speed end-mill and subsequently not touched along the interfacial edge. Touching the milled surfaces caused noticeable degradation in the measured bond strength possibly due to residual skin oils [8]. Cutting the sheets from the same cast plate prevented thickness variations at the interface.

The two plates were finished to the same width so that when they were placed together in a mold, the sheets were in compression by an overall strain of 0.1 percent in the direction transverse to the interface. The mold consists of two $25 \mathrm{~mm}$ thick by $300 \mathrm{~mm}$ square $17-4 \mathrm{PH}$ stainless steel plates, called platens, that sandwich the material, and of $10 \mathrm{~mm}$ thick side plates that compress and constrain the polymer from extruding out along the edges between the platens. These platens were lapped flat to less that $10 \mathrm{~nm} / \mathrm{mm}$ and then polished smooth with diamond grit as fine as $1 \mu \mathrm{m}$.

The entire assembly was surrounded with a woven glass breather material (a medium porous to gasses). The breather material was in turn surrounded with a thin sheet of impermeable nylon. The interface between the breather and bagging material was vented to a small piston, oil vacuum pump. This assembly was placed in a hydraulic press possessing temperature controlled platens. This whole operation was performed in a room that was cleaned to prevent dust contamination and the vacuum pump was in a remote room to prevent possible exposure of the surfaces to oil vapors.

With the specimen in the mold, a vacuum (29 inches of $\mathrm{Hg}$ ) was established for $10^{4} \mathrm{~s}$ after which the seal on the system was verified. With the vacuum maintained, the temperature of both the upper and lower surfaces of the mold was increased to a 'healing' temperature above the 
glass transition temperature of the PMMA and the pressure between the platens was increased to $100 \mathrm{psi}$ above ambient. The temperature of the polymer was raised to the healing temperature in less than $10^{3} \mathrm{~s}{ }^{*}$ The temperature and pressure was held constant from one to five hours. After this period the hydraulic load was removed and the specimen was allowed to cool to room temperature. Once the assembly was at room temperature, the vacuum was slowly removed and the specimen very carefully separated from the mold platens.

\subsubsection{Characterization}

To ascertain the nature of the bond, a selection of these samples were very carefully machined into $7.3 \mathrm{~cm}$ square, nearly standard ASTM compact fracture specimens. The specimens were standard in their planar geometry but their thickness was sub-standard [13]. The compact fracture specimens were cut from two places on the plate. One sample was cut from a virgin region unaffected by the sintering procedure. A second specimen was cut so that the sintered interface was coplanar with the notch in the compact fracture specimen. The notch was cut using a slitting saw. The tip of the notch was filed to a radius of approximately $0.5 \mathrm{~mm}$ and a true crack started with the impact of a razor blade.

The blade-started crack was fatigue loaded with a $1.0 \mathrm{~Hz}$ sinusoidal waveform until the crack propagated to a total length of $40 \mathrm{~mm}$. Once at this length the end displacement was slowly increased and the load recorded until the crack started to propagate. The maximum recorded load was taken as a measure of the strength of the interface. The crack always propagated straight and in a brittle fashion. In addition, once crack propagation was initiated, the crack position and load never demonstrated any noticeable discontinuities, thus lending confidence to the uniformity of the bond. The same tests were repeated on specimens manufactured out of identical virgin material (without the interface). The results of these tests are shown in Fig. 1.

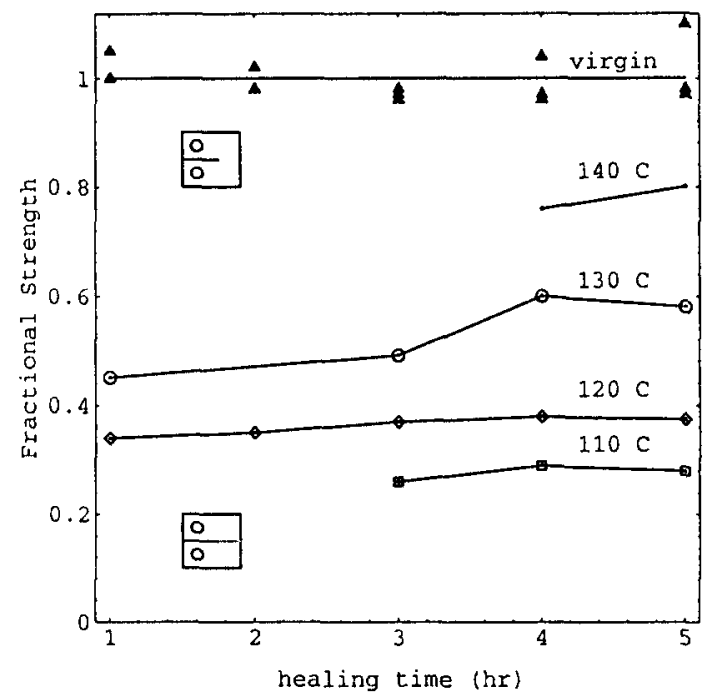

Fig. 1. Strength as a function of healing time and four molding temperatures for virgin and interfacial PMMA specimens. Here the fractional strength is the measured fracture load divided by the nominal fracture load of PMMA.

\footnotetext{
* The 'temperature of polymer' was actually determined from thermocouples embedded in the platen surfaces. A calibration was performed by simultaneously embedding a thermocouple in the middle of the cast polymer that showed this to be an accurate statement for these time scales.
} 
As one expects, increasing the molding temperature or healing time increases the strength of the interface. Higher temperatures and longer times increased the interpenetration of the molecule chains across the interface with a corresponding increase in its strength. Tests at a higher temperature of $150^{\circ} \mathrm{C}$ were performed and had fractional strengths very close to the virgin specimens, however, the interface was either not distinguishable under the microscope from the parent material, or achieving crack propagations along the interface was not accomplished (i.e. a near completely rehealed interface was achieved). Because it was not clear that the crack was propagating along the interface, the results at $150^{\circ} \mathrm{C}$ are not included in Fig. 1. Interfaces at lower healing temperatures of $105^{\circ} \mathrm{C}$ and $100^{\circ} \mathrm{C}$ were also produced but since the specimens were unsupported in various phases of construction they were invariably broken before the strength measurements.

The interfaces were illuminated in transmission and examined under a microscope. The interfaces appeared as a dark band $0.01 \mathrm{~mm}$ thick that interrupted the normally transparent properties of PMMA. In addition after coating one surface with a vapor deposited layer of aluminum, the true out-of-plane deformation of the sample while under load was measured using a He-Ne Twyman-Green interferometer. Shown in Figs. 2 and 3 are the out-of-plane deformations near the crack tips, at the anterior surface of the sheet, of both a virgin and the weakest interfacial specimen near the critical fracture loads. The rational for making this measurement and a more extensive interpretation of these interferograms will be given in the following section. However, here we make some observations pertinent to the strength measurements in Fig. 1.

One difference between the virgin and interfacial specimens is the spatial density of fringes. The virgin specimen exhibits approximately five times the number of fringes over the interfacial

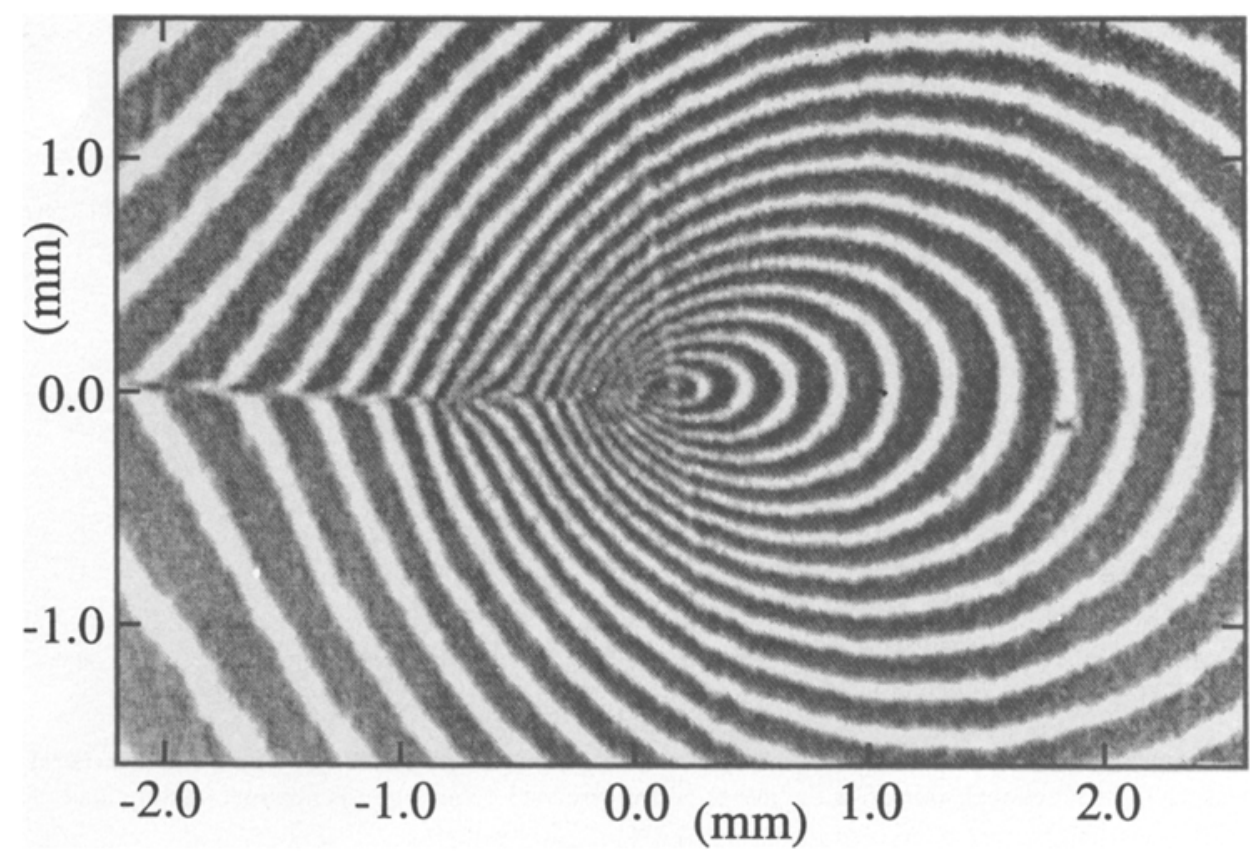

Fig. 2. Out-of-plane displacement contours at the tip of a stationary crack in a virgin specimen of polymethylmethacrylate. A near compact fracture specimen geometry, $4.5 \mathrm{~mm}$ thick, at a load of $135.0 \mathrm{~N}$. Surface contours represent displacements of $312 \mathrm{~nm}$. 


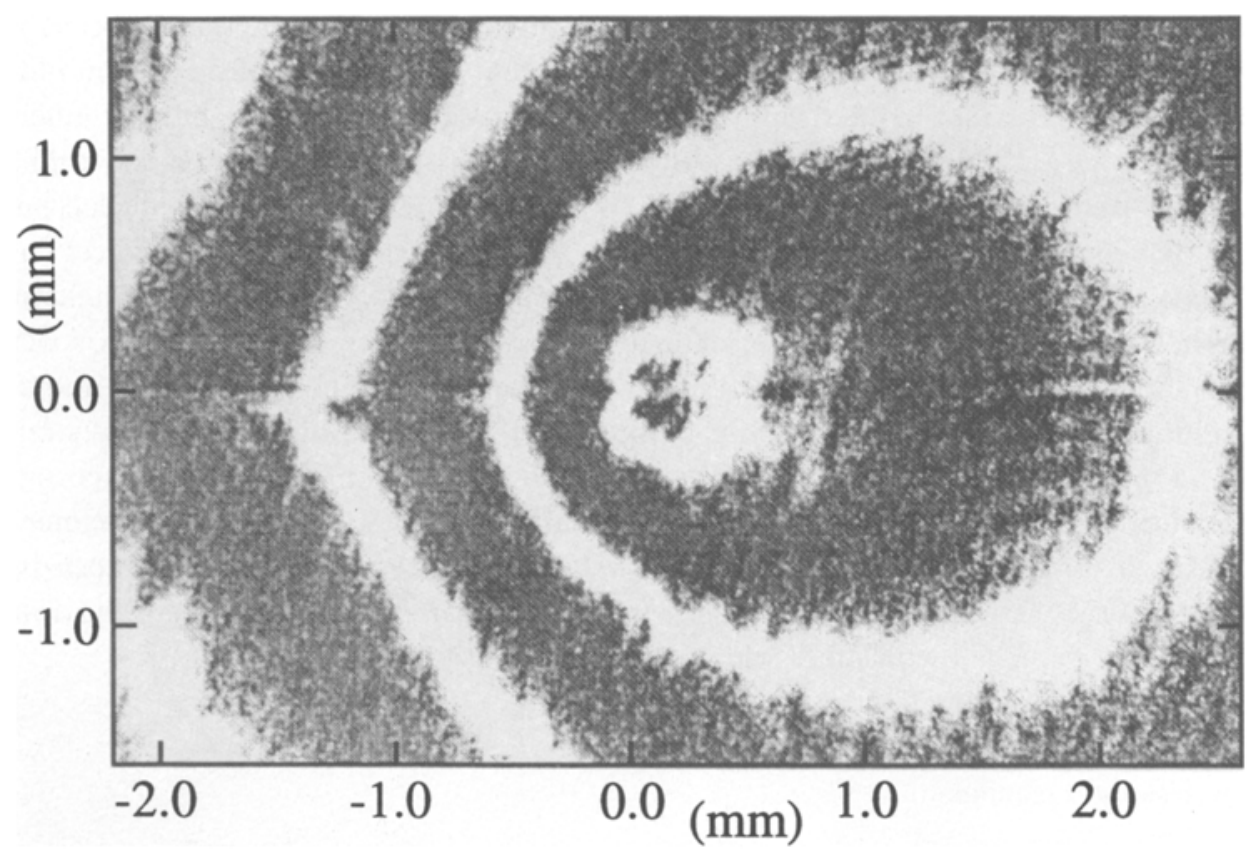

Fig. 3. Out-of-plane displacement contours at the tip of a stationary crack in an interfacial specimen of polymethylmethacrylate. The interface was healed at $110^{\circ} \mathrm{C}$ for 3 hours. Specimen geometry and contour separation as in Fig. 2, at a load of $26.9 \mathrm{~N}$.

sample because the virgin interferogram is taken at a commensurately higher load. Under ideal circumstances the interferograms should be symmetric with respect to the crack. Outside the field of view of Fig. 2 there is a pronounced lack of symmetry. At higher loads, the compact fracture specimen used in this investigation tended to warp asymmetrically out-of-the-plane. The virgin specimen displays an almost vertical scratch just preceding the crack. The healed interfacial specimen shows a similar horizontal feature. In the latter case this is a small ridge at the intersection of the sintered interface and the free surface. In other words, the healed interface initially appears on the surface as a very slight off-set, and of a magnitude similar to that of a typical scratch.

At distances far from the crack tip, the fringe contours appear very similar. That is, the interface does not demonstrate a pronounced out-of-plane deformation. Here 'far' corresponds to a distance of 0.5 times the plate thickness. This is within a region where plane approximation to the out-of-plane surface deformation is poor because the geometry and deformation is truly three-dimensional [14]. Closer to the crack tip the deformation contours of the interfacial specimen demonstrate a cusp that is not apparent in the virgin material. At the tip, the crack front for the virgin specimen had a slight curvature, while for the weaker interfaces, the crack front appeared straight. This cusped feature could also be the result of the initial small offset of the interface. These interferograms demonstrate that the difference in material response between the two types of specimens, at least at the surface, is very small in a region close to the crack front.

\subsection{Array of holes}

It has been observed that cracks do not propagate in a steady manner at high velocities in PMMA [15] but in approximately discreet periodic steps [16]. These steps are large in that they 
can have lengths on the order of a millimeter. The imperfectly healed interface is perhaps more analytically tractable from an elasticity standpoint in that it is intended as a slight modification of the otherwise pristine material. However, since truly dynamic cracks have a much more violent and large scale nature, perhaps a periodic array of small holes placed on a millimeter scale pitch would in some sense be equivalent to (or indistinguishable from) a healed interface. In addition some aspects of a crack propagating in the presence of preexisting discreet flaws can be examined. Thus, for purposes of comparison a second set of specimens were manufactured by drilling holes of $0.25 \mathrm{~mm}$ and $0.50 \mathrm{~mm}$ diameters in front of the crack at various spacings in integer increments of $0.50 \mathrm{~mm}$. These hole sizes and spacings were manufacturable and allowed the resulting area fraction* to have a range approximating the measured fractional strength of the healed interface. These holes were drilled along a line $10 \mathrm{~cm}$ long. For a single specimen this involved drilling hundreds of holes using a tiny and fragile drill bit. These specimens were manufactured using a numerically controlled milling machine to perform the repetitive hole drilling operation. This single array of holes is a step toward studying the behaviour of a material that is damaged with more widely distributed discreet flaws.

\section{Experimental technique}

The goal of these experiments is to approximate an impulsively loaded infinite plate and observe the resulting behavior of the material surrounding an initial crack. Since the material is being altered on a very fine scale, a measurement with high spatial resolution is needed to compare the effects of the modification on the response of the crack. In addition, as shown between Figs. 2 and 3 the effect of the modification may only be evident at the crack tip; thus necessitating an experimental technique that can observe the crack tip. This work complements previous dynamic caustic experiments [9] ${ }^{\dagger}$. It is also a direct extension of an earlier interferometric study in that the method of dynamically loading the specimen and the measurement equipment employed here are similar [16].

\section{I. Specimen construction}

The dynamic specimens were constructed from pellets of PMMA that were cast, and slowly annealed into smooth flat sheets nominally $300 \mathrm{~mm}$ square by $4.5 \mathrm{~mm}$ thick as described in Section 2. For specimens incorporating weakened interfaces the rehealing technique previously described was also employed.

To provide a testing time free of reflected waves from the boundaries of approximately $100 \mu \mathrm{s}$ the sheet was left whole and a notch was cut to a length of $19 \mathrm{~cm}$. This specimen geometry is shown in Fig. 4. These sheets were notched using a straight end mill and a file. A starter crack was initiated using an impulsively loaded razor blade wedged in the filed notch. The anterior surface was vacuum coated with pure aluminum to give a specularly reflective surface.

\footnotetext{
* The area of the remaining material on the plane in front of the crack divided by the original area. ' Since the work in [9] the validity of the caustic method has been examined exhaustively. In contrast to situations involving plasticity and reflection, the transmissive caustic method is sensitive to well established in-plane field components [17] and provides excellent data for small deformation brittle materials. Aspects of this question of validity of the measurement will be discussed later in this section.
} 
As documented previously [16] the crack flanks were impulsively loaded (with a rise time of $20 \mu \mathrm{s}$ ) by discharging a high voltage capacitor bank through a triple-wound copper strip by means of a mercury switch. The width of the milled notch was set to allow a snug fit of the loading coil between the crack flanks without pre-loading the crack.

\subsection{Measurement technique}

The out-of-plane displacement of the reflective surface was measured using a Twyman-Green interferometer [18] and a high-speed rotating mirror camera, with illumination provided by a Spectra-Physics model 166, $5 \mathrm{~W}$ Argon Ion laser tuned to $514 \mathrm{~nm}$ with $15 \mathrm{~ns}$ light pulses generated from a Bragg cell. The laser light was expanded, filtered and collimated to a $50 \mathrm{~mm}$ diameter through a $30 \mathrm{X}$ microscope objective, a $12.5 \mu \mathrm{m}$ diameter pin hole, and a Nikon $200 \mathrm{~mm} \mathrm{f} / 4$ IF lens. The collection optics include a Nikon $200 \mathrm{~mm} \mathrm{f} / 2 \mathrm{IF}$ lens, a $50.8 \mathrm{~mm}$ focal length field lens at the intermediate focal plane, and a Nikon TC-14B teleconverter and a Nikon $200 \mathrm{~mm}$ IF lens between the intermediate focal plane and the final image. The film used was Kodak T-Max 400 processed with Kodak T-Max developer. A schematic of this apparatus is shown in Fig. 5.

The finished specimen with the loading coil inserted, was placed in an insulated enclosure that had several small apertures to allow passage of the light from the interferometer and the current from the capacitor bank. This enclosure protects the surrounding instrumentation by containing debris from the specimen and loading coil. The specimen was aligned so that the normal to the anterior surface just in front of the crack tip was parallel with the laser. The optics were focused on this reflective surface. Photographs were recorded every $20 \mu$ s starting at $100 \mu$ s before the initiation of the loading $[11,16]$. The average velocity of crack propagation was determined by examining the location of the crack in successive frames.

\subsection{Twyman-Green interferometry discussion}

A very important aspect of this study is the demonstration that the manufacturing process produced specimens in which the global properties (moduli, density, wave speeds) remained

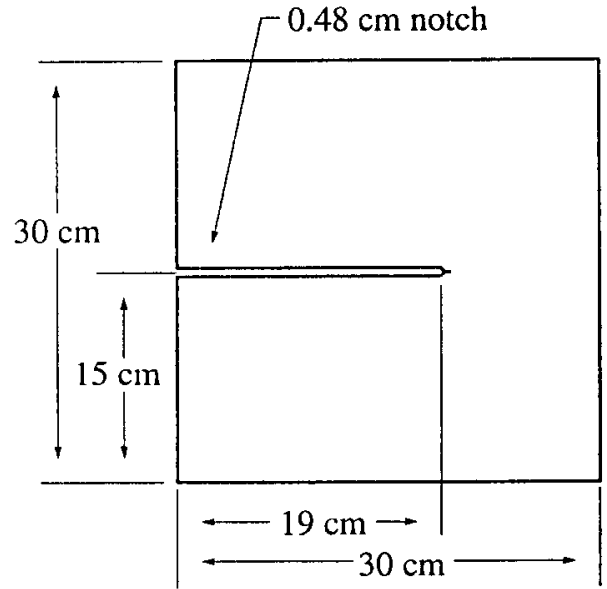

Fig. 4. PMMA specimen geometry for dynamic tests.

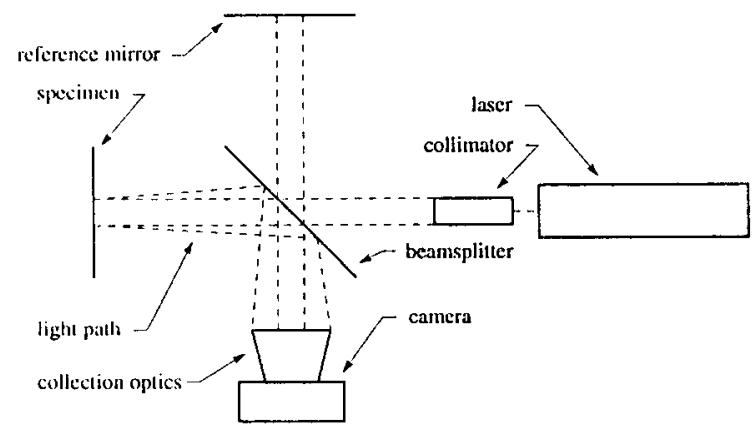

Fig. 5. Schematic of the Twyman-Green interferometer to measure out-of-plane displacement [18]. 
unaltered while the physical strength of the interface was 'controlled'. The only means we have available to monitor the continuity of the elastodynamic field at the crack tip is through high resolution, high speed, interferometric surface displacement measurements. Moreover, the effects of the presence or absence of small flaws as indicated by surface waves emitted by the moving crack tip requires careful analysis of the optical records. For these reasons it is appropriate to discuss the advantages - and limitations - of the optical method employed.

There are numerous optical methods to measure the behavior of a solid in the vicinity of a crack. Methods range from measuring a single quantity e.g. shadow-graphic methods $[19,20]$ and Fourier-filtered caustics [21], to those that observe entire fields, e.g. photoelasticity [22], speckle [23], diffractive [24], and holographic methods [25]. Some measurement techniques have clear restrictions to materials that have a special property such as transparency for transmitted caustics or birefringence for photoelasticity. Addressing some of the attributes of Twyman-Green interferometry in comparison with these more established fracture measurement techniques will explain the rational for selecting this type of measurement method.

There is generally a trade-off between spatial and temporal resolution amongst measurement techniques [18]. In situations where the deformation field is not well established, it is preferable to measure spatial information at the expense of measurement frequency. For instance photoelastic observations [26] clearly indicate the spatial state of the crack field and the necessary interpretation requirements over a more filtered, but temporally superior, technique such as caustics [27]. In dynamic situations light efficiency is typically a concern and techniques that incorporate light diffusive elements such as diffractive [24] or diffusive [25] surfaces are at a disadvantage.

Twyman-Green interferometry as used here measures the out-of-plane deformation field on a surface. This method has some distinctive attributes. A specular reflective surface is employed leading to high light efficiency. Also, since the optics are particularly simple* a specimen can be designed to allow measurements right up to the crack tip with very fine spatial resolution. This spatial sensitivity is of paramount importance in this investigation to detect any evidence of an effect of small initial flaws at the crack tip. Here the thickness was maximized to maintain the flatness of the specimen with the constraint of the numerical aperture of the collection optics. Measurement of the deformation of the surface has the desirable attribute that it is spatially precise in that it avoids through the thickness averaging as is the case with transmission techniques [18].

However, many of these favorable attributes have some corresponding disadvantages. Interpretation of the out-of-plane-displacement near the tip of a crack is in its infancy, especially under dynamic conditions. As is usually assumed for work with reflected caustics, there is not a simple $K$-dominant field surrounding the crack $[26,28]$. Three dimensional and transient terms from the loading need to be taken into account making direct interpretation of the measurements a non-trivial task. Further, since this measurement technique is solely sensitive to the out-of-plane deformation of the outside surface, normal planar approximations near the tip are not adequate $[29,30]$. In the case of virgin PMMA the surface displacement is even worse in that the crack does not propagate steadily at fracture speeds above $0.33 \mathrm{~mm} / \mu \mathrm{s}$ [15]. This results in an additional transient phenomenon that is typically not incorporated in any analyses.

\footnotetext{
* Between the camera and the specimen is a single partially reflective surface from the beam-splitter.
} 
Twyman-Green interferometry is used here in spite of these shortcomings for its sensitivity and spatial resolution at the crack tip.

One difficulty with this interferometric technique had been that any initial surface warpage was carried through to every subsequent image. As can be seen in the data previously presented by Washabaugh and Knauss [16] this technique was adequate to show surface waves, however direct comparison between images with different initial surface warpages was problematical. One option is to slowly anneal each specimen for weeks to maintain an interferometrically flat surface once it is removed from the mold [11]. Another option is to simply subtract the initial deformation from the subsequent images.

One difficulty with this subtraction procedure is that the interferometry measurement done here is not one-to-one invertible. The displacement of the surface of the specimen is not measured directly. The phase of the light (i.e. $\phi$ ) returning from the specimen is displaced in proportion to the deformation of the surface. This light interferes with a coherent reference signal (here a plane wave) resulting in a sinusoidal variation of the electric field strength (i.e. $\left.E=E_{0} \sin (\phi)\right)$ of the light as function of the difference in phase. The square of the electric field (i.e. $I=E^{2}$ ), or the intensity, is actually measured using film. Typically film also has a nonlinear response to intensity. Thus Figs. 2 and 3 approximately represent the sine squared of the deformation of the surface. Inverting this transcendental function leaves an uncertainty in the phase order, and in general regions where the shape of the sürface is more difficult to interpret (e.g. saddle points [16]) can cause additional ambiguities.

A typical response to this problem of phase ambiguity is to rotate the specimen so that the resulting change in phase is biased to be monotonic. Since the slope near the crack tip is already limited by the angular collection capability of the optics, rotating the specimen is not an option if the full field is to be measured. Another possibility is to photograph the process with a second camera system that is identical to the first except that there is a quarter-wave plate in the optical path. The wave plate retards the phase of a parallel set of images but would require the expense of a second high speed recording system.

Here certain tests yielded interferograms with a small number of saddle points. The curvature of the initial surface could be ascertained before the test by rotating the reference mirror. It was also assumed that the deformation at the crack tip during the loading was inward. Using this information a mostly automatic sequence of computer programs was used to process the images. These programs [31] balance the average intensity throughout the image to correct for non-uniform illumination, make first and second order corrections for film response nonlinearity [32], invert the intensity to the magnitude of deformation (i.e. phase), and locate fringe centers. The direction of the change in phase was entered manually. For relatively simple images this process allowed the initial deformation to be subtracted yielding the true displacement of the surface.

\section{Experimental results}

A typical enlargement of a processed photograph from a test of a virgin specimen, at $60 \mu \mathrm{s}$ after the start of loading, is shown in Fig. 6. The initial unloaded shape of the specimen has been subtracted. Based on the data at $40 \mu$ s and $80 \mu$ s the crack is moving at an average velocity of $0.52 \mathrm{~mm} / \mu \mathrm{s}$. The interferogram has two superimposed displacements. First there is the general indentation near the tip (located at 0,0 ) due to the Poisson contraction of the material from the 


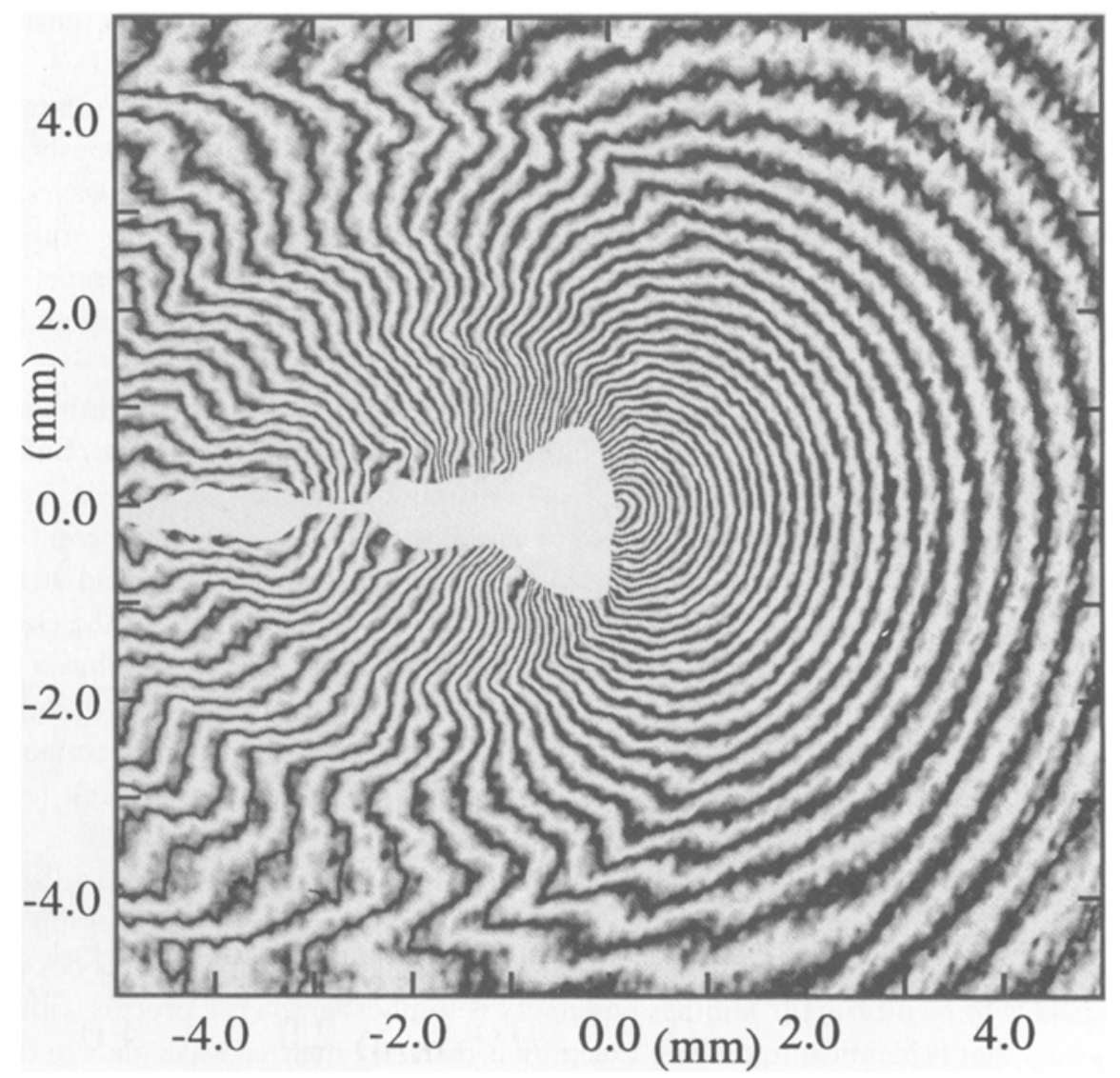

Fig. 6. A corrected interferogram of the out-of-plane surface displacement of a crack moving at an average velocity of $0.52 \mathrm{~mm} / \mu \mathrm{s}$ in a virgin $4.6 \mathrm{~mm}$ thick plate of polymethylmethacrylate. Contours represent a $257 \mathrm{~nm}$ change in elevation.

mode-I loading of the crack flanks. The second deformation is a ripple pattern radiating outward from near the crack tip. Also, since the initial deformation has been subtracted, there is a slight asymmetry in the displacement about the crack path, far from the tip. This is probably due to a slightly asymmetric far-field load. The white region along the crack flanks is a 'shadow' spot (processed to white), a consequence of the angular aperture limitations of the collection optics.

The crack is propagating through the macroscopically amorphous material at about one-half of the Rayleigh wave velocity. At this speed, waves are radiated away from the moving crack tip with approximately the shear wave velocity. These surface ripples are generated periodically at a rate of one every 0.8 microseconds, implying that a surface wave is produced for every $0.4 \mathrm{~mm}$ of crack advance. The surface morphology of this fracture process has a corresponding simple $0.4 \mathrm{~mm}$ periodic, approximately straight, ripple structure that is on average parallel with the plate normal [16].

A typical enlargement of a processed photograph of a rehealed interfacial specimen at $60 \mu \mathrm{s}$ after the start of loading is shown in Fig. 7. Again the initial shape of the specimen has been subtracted. Based on the crack location at $40 \mu$ s the crack is moving at an average velocity of $0.86 \mathrm{~mm} / \mu \mathrm{s}$. (At $80 \mu \mathrm{s}$ the crack left the field of view). As in the virgin specimen there is a general Poisson contraction toward the crack tip. Again there are small ripples, though now barely 


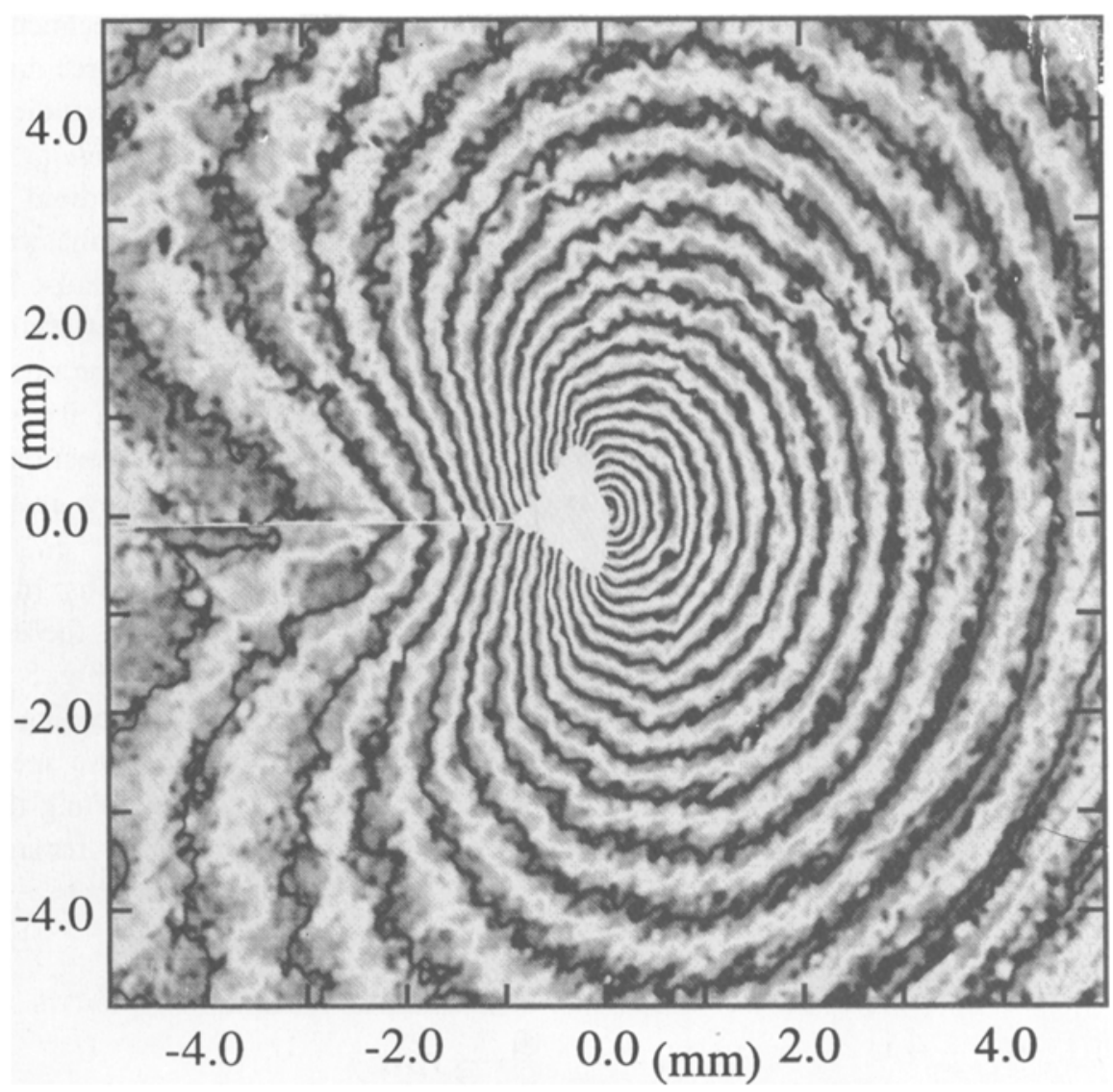

Fig. 7. A corrected interferogram of the out-of-plane surface displacement of a crack moving at an average velocity of $0.86 \mathrm{~mm} / \mu \mathrm{s}$ in an interfacial $4.7 \mathrm{~mm}$ thick plate of polymethylmethacrylate. The crack is propagating along an imperfectly sintered interface that is $60 \%$ of the strength of the virgin material. Contours represent a $257 \mathrm{~nm}$ change in elevation.

discernible, radiating from the crack. The far-field fringes are generally symmetric about the crack path.

Both images show evidence (of differing degrees) of non-steady crack propagation. The temporal frequency of these measurements are about an order of magnitude too course to trace the details of a single period in the fracture process. However, some additional information about how the crack proceeds can be gleaned from the detailed spatial resolution. In particular the crack appears to move and generate this periodic behavior with an oscillation in the propagation direction. The surface ripples radiating from the crack tip appear symmetric with respect to the average crack propagation direction. This is suggestive of a jerking, accelerating-decelerating, or discontinuous, process with a constant crack direction, as opposed to a crack that is propagating with a rapid oscillation in direction (e.g. non-planar motion [6]). The main difference, with regard to this nonsteady crack behavior, between these two tests is that in the interfacial case the severity is greatly reduced over the virgin specimen. In effect, the weakened interface has nearly suppressed the non-steady behavior, presumably by eliminating the nucleation and growth of micro-flaws preceding the crack. 
Moreover, the general Poisson contraction near the tip of the two specimens can be compared. Several numerical studies have been performed with regard to the three dimensional nature of the deformation field near a crack tip [e.g. 14, 30, 33, 34]. These studies have in common that the cardioid out-of-plane displacement field predicted by the singular term in a planar approximation (the $K$-field) is not present. Instead the cusp of the cardioid along the crack flanks is 'relaxed' and compares loosely with Figs. 6 and 7 (e.g. see Krishnaswamy et al. [29], Fig. 9c). An exact comparison of these previous studies is not expected because the cracks here are transient, rapidly and non-steadily propagating. Except for the severity of the surface ripples and the difference in fringe density (owing to the reduced strength of the interface) the virgin and interfacial out-of-plane displacement fields are very similar. The fringes of the interfacial specimen are slightly more compressed in the crack propagation direction than for the virgin specimen. This compression of the fringes would be expected as a result of the increased fracture speed [35].*

The tests were repeated with specimens possessing varying interfacial strengths. In addition, tests using specimens with an array of holes were performed. The velocity of the crack as a function of a property of the material in front of the crack is summarized in Fig. 8. Here the Rayleigh wave speed is taken as $1.04 \mathrm{~mm} / \mu \mathrm{s}$. It is clear that as the material preceding the crack front becomes weaker the crack runs faster and approaches the Rayleigh wave speed of the virgin material. The Rayleigh wave speed is not actually achieved, thus supporting the notion that this speed is a kinetic limit on the crack propagation behavior (see earlier remarks in the Introduction and [5]).

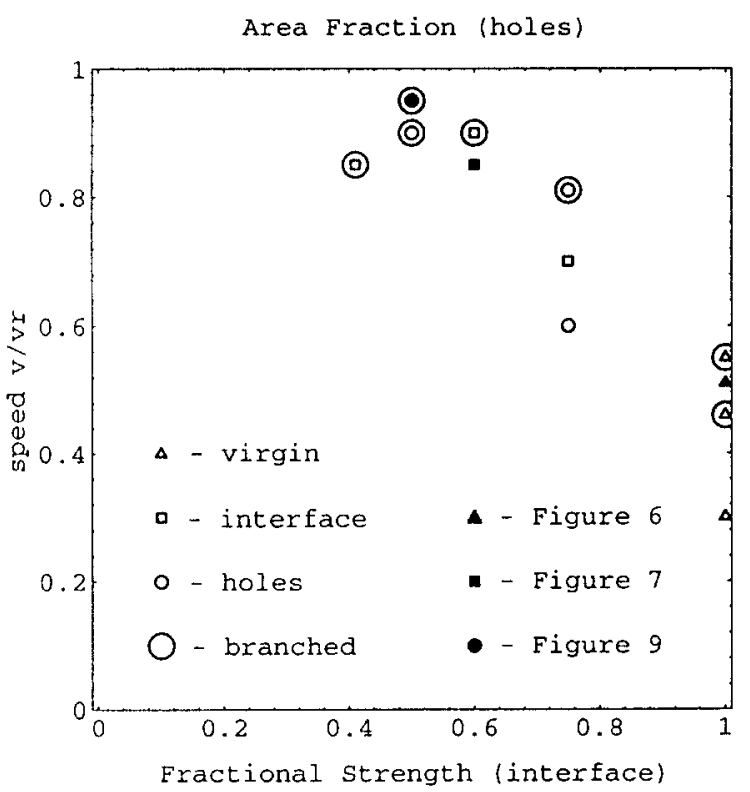

Fig. 8. Dependence of the crack velocity on the property of the material (i.e. fractional strength or area fraction) preceding the crack front.

* The fracture morphology of PMMA at these velocities suggests the crack front is nearly parallel with the plate normal $[15,16]$. This 'compression' of the fringes or increase in slope at the crack tip has been predicted for a crack front that is nearly parallel to the plate normal [35]. 
The circle surrounding a symbol indicates that within the field of view more than one crack was propagating. At higher load levels the crack would frequently and almost immediately branch from the initial crack. In some cases however, the crack would propagate a small distance from the initial crack before branching. The holes were especially troublesome in that a crack intersecting a hole near the point of initiation almost invariably resulted in multiple cracks. However, it is of particular interest to note that all branches were established very near the point of initiation of crack propagation; none of the cracks propagating along the interfaces produced further branches once the crack left the region of initiation.

Figure 8 clearly shows that as the local strength of the material decreases, the fracture velocity increases. Figures 6 and 7 demonstrate that the mean out-of-plane displacement field is not appreciably altered by the increased crack velocity and there is no noticeable deformation caused by the introduction of the weakened interface. In effect the interface has been enfeebled sufficiently to increase the speed of the crack, yet subtly enough to not disturb the out-of-plane deformation field.

Suppression of the non-steady behavior by itself is not necessary to increase the speed of the crack. As a demonstration we show Fig. 9 where a crack is running near the Rayleigh wave speed with some dramatic non-steady behavior. This is a view of a crack at $60 \mu$ s after the start of the loading pulse running along an array of holes. Here the initial surface deformation remains (i.e. an unprocessed image). In addition inside the displayed field of view two other (branched) cracks (roughly at coordinates $(-11,-13)$ and $(-14,12)$, and at inclinations of $\left.+/-\frac{1}{4} \pi\right)$ are propagating. Here in a single specimen, one crack is running near the Rayleigh wave velocity along the interface while the other two cracks travel at approximately one-half this velocity because they are propagating through pristine material. This behavior was also typical for the rehealed specimens.

Figure 9 also demonstrates a wealth of features typical to the interferograms in this study. The crack running along the array of holes has shear waves visible on the surface radiating away from the moving crack. The spacing of the largest of these waves is consistent with the millimeter hole spacing, i.e. approximately one wave generated for each hole. However, there are also smaller amplitude ripples on a sub-millimeter pitch between the larger waves. These smaller ripples cloud the precise one-to-one correspondence of waves to holes. These smaller waves are likely produced by the fracture of the material between the holes. Support for this statement is shown in the same interferogram. The two branched cracks moving through the pristine material have very similar small amplitude periodic waves on a sub-millimeter pitch radiating from the crack.

In Fig. 9 the large amplitude waves and their multiple sources (i.e. from more than one crack) coupled with the initial surface deformation demonstrate some of the limits of the current apparatus and the difficulties in interpreting these interferograms. The large amplitude waves near the main crack show kinks in the surface where the fringe continuity and contrast is lost. In addition the intersecting wave fields from the cracks result in many multiple regions where the displacement order of the fringes is ambiguous.

For the interfacial specimens the increased crack velocity was also accompanied by a more steady crack motion as indicated by the reduced severity of shear waves radiating from the crack tip. However in the specimens with the array of holes, as indicated by the sharp wave fronts radiating from the crack tip, the crack velocity was augmented while increasing the amplitude of the non-steady crack propagation behavior. This indicates that for a planar interface the overall 


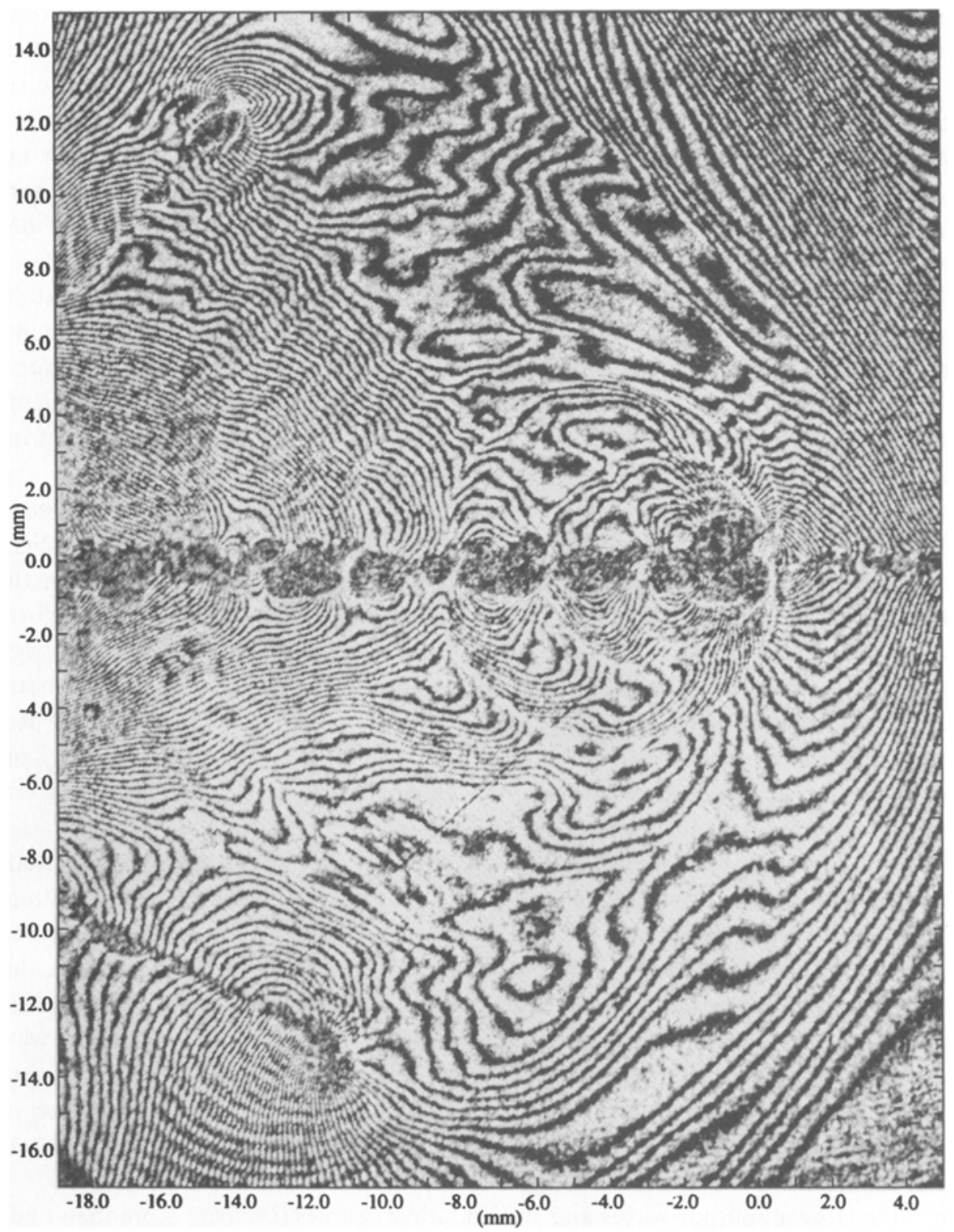

Fig. 9. An unprocessed (initial deformation remains) interferogram of the out-of-plane surface displacement of a crack moving at an average velocity of $0.95 \mathrm{~mm} / \mu \mathrm{s}$ in a specimen with an array of 0.5 diameter holes on $1.0 \mathrm{~mm}$ centers in a $4.5 \mathrm{~mm}$ thick plate of polymethylmethacrylate. Contours represent a $257 \mathrm{~nm}$ change in elevation. Two other cracks are moving on either side of the crack running along the interface. A scratch on the film (at an angle of $\sim \frac{1}{4} \pi$ ) is also visible.

effective strength, and not the detailed spatial distribution of the enfeeblement, primarily governs the crack speed. A similar statement can be made for crack branching. Except near initiation, the cracks in the weakened specimens can run at velocities approaching the Rayleigh wave speeds without branching. The persistent branching near initiation indicates that the initial crack (i.e. shape, length, proximity to loading coil) needs further refinement. However, once the propagating crack leaves this initial transient region, branching does not occur inspite of the widely varying macroscopic nature of the interfaces. 


\section{Summary remarks}

Local enfeeblement of the intrinsic strength of an amorphous material increases the maximum crack speed to near the Rayleigh wave speed. The crack velocity is augmented while the typical non-steady behavior is reduced but the general local displacement field is maintained when compared to a crack propagating through a pristine material of higher intrinsic strength. These observations are consistent with the suppression of the micro-cracks observed by Ravi-Chandar and Knauss [9]. Weakening the material in front of the crack with a series of discreet flaws also increases the average crack speed to near the Rayleigh wave speed while maintaining the non-steady crack propagation behavior. For comparable crack speeds, the test results indicate that the area fraction in this case is roughly equal to the fractional strength of a healed interface. We thus deduce that linear elastodynamics correctly predicts the dynamic crack propagation speed in solids possessing properties (e.g. low intrinsic material strength) that suppress multiple micro-crack formation so as to propagate a single crack front.

Moreover, we note that there is an absence of crack branching for cracks running along a rehealed interface, and in the presence of large discreet flaws. Specifically, the absence of branching for the materials with a weak plane moving near the Rayleigh wave speed contradicts the argument that branching is principally a kinetic effect as suggested by Yoffe [36]. This, velocity-detached view of branching is further reinforced by the observations that branching occurred primarily at crack initiation while the crack had not established its propagation direction and velocity. An explanation consistent with these observations is that for the enfeebled specimens the branching is suppressed because there is an absence of micro-cracks unstably interacting with the main crack [9].

A final observation relates to implications of this study for the dynamic fracture of bi-material interfacial bonds: In that case one should expect that separation speed should readily approach the Rayleigh wave speed of the slower of the two materials because adhesive bond strength in such situations is typically less than optimal. Indeed, it appears possible that for suitably weak interfaces, separation speeds could attain values intermediate to the Rayleigh speed of the two materials (i.e. become supersonic with respect to the slower material) and even approach the Rayleigh speed of the faster solid in the limit of zero bond strength.

\section{References}

1. B. Cotterell, Applied Materials Research 4 (1965) 227-232.

2. L.B. Freund, Journal of the Mechanics and Physics of Solids, Pt. I-20, 129-140; Pt. II-20, 141-152; Pt. III-21, 47-61; Pt. IV-22, 137-146 (1972-1974).

3. O.S. Lee and A.S. Kobayashi, in ASTM STP 868, M.F. Kanninen and A.T. Hopper (eds.) (1985) 431-450.

4. A. Shukla and J.W. Dally, Experimental Mechanics (1983) 298-303.

5. W.G. Knauss and K. Ravi-Chandar, International Journal of Fracture 27 (1985) 127-143.

6. H. Gao, The Journal of the Mechanics and Physics of Solids 1 (1993) 457-486.

7. J. Field, Contemporary Physics (1971) 1-31.

8. W.G. Knauss and O.S. Lee, Experimental Mechanics 29 (1989) 342-345.

9. K. Ravi-Chandar and W.G. Knauss, International Journal of Fracture, Pt. I-25, 247-262; Pt. II-26, 65-80; Pt. III-26, 141-154; Pt. IV-26, 189-200 (1993).

10. S. Wu and A. Chudnovsky, International Journal of Fracture 59 (1993) 41-52.

11. P.D. Washabaugh, Ph.D. dissertation, California Institute of Technology (1990).

12. Aldrich Chemical Company, 940 West Saint Paul Avenue, Milwaukee, Wisconsin 53233, USA, Product \#18, 224-9. 
13. ASTM E 399-74, Part 10 (1974).

14. W.S. Burton, G.B. Sinclair, J.S. Solecki and J.L. Swedlow, International Journal of Fracture 25 (1984) 3-32.

15. J. Fineberg, S.P. Gross, M. Marder and H.L. Swinney, Physical Review B 45 (1992) 5146-5154.

16. P.D. Washabaugh and W.G. Knauss, International Journal of Fracture 59 (1993) 189-197.

17. C.R. Schultheisz, Ph.D. dissertation, California Institute of Technology (1991).

18. R.D. Pfaff, Ph.D. dissertation, California Institute of Technology (1991).

19. J. Beinert and J.F. Kalthoff, in Mechanics of Fracture, G. Sih (ed.), Noordhoff (1981) 281-330.

20. P.S. Theocaris, Applied Optics 10 (1971) 2240-2447.

21. K.S. Kim, ASME Journal of Applied Mechanics 52 (1985) 291-297.

22. A.A. Betser, A.S. Kobayashi, O.S. Lee and B.S.J. Kang, Experimental Mechanics (1982) 132-138.

23. W.T. Evans and A. Luxmoore, Engineering Fracture Mechanics (1974) 735-743.

24. H.V. Tippur, S. Krishnaswamy and A.J. Rosakis, International Journal of Fracture 52 (1991) 91-117.

25. T.D. Dudderar and R.O. Regan, Experimental Mechanics (1971) 49-56.

26. C. Taudou, S.V. Potti and K. Ravi-Chandar, International Journal of Fracture 56 (1992) 41-59.

27. K.S. Kim, Journal of Applied Mechanics 52 (1985) 585 592.

28. K. Takahashi and K. Arakawa, Experimental Mechanics (1987) 195-200.

29. S. Krishnaswamy and A.J. Rosakis, Journal of Applied Mechanics 58 (1991) 87-94.

30. Ibid, 95-103.

31. P.D. Washabaugh, personal notes: Image processing programs for fringe analysis (1992).

32. Eastman Kodak Company, Kodak Publication No. F-5, Rochester, NY (1987).

33. T. Nakamura and D.M. Parks, Three-dimensional field near the crack front of a thin elastic plate, presented at 24th Annual Meeting of the Society of Engineering Science, September 21-23, 1987, Salt Lake City, Utah.

34. R.S. Barsoum and T.K. Chen, International Journal of Fracture 50 (1991) 221-237.

35. P. Gudmundson and S. Ostlund, Journal of Applied Mechanics 57 (1990) 112-116.

36. E. Yoffe, Philosophical Magazine 42 (1951) 739. 